\title{
Rifalazil Retains Activity Against Rifampin-resistant Mutants of Chlamydia pneumoniae
}

\author{
David M. Rothstein, Robert J. Suchland, Minsheng Xia, Christopher K. Murphy, \\ Walter E. Stamm
}

Received: February 16, 2008 / Accepted: July 18, 2008

(C) Japan Antibiotics Research Association

\begin{abstract}
Rifampin-resistant mutants of the obligate intracellular pathogen Chlamydia pneumoniae were isolated and characterized, including strains that contained multiple mutations in the $r p o B$ gene encoding the rifampin binding site. The highest MIC of rifampin against a mutant strain exceeded $100 \mu \mathrm{g} / \mathrm{ml}$, whereas the highest MIC of rifalazil was $0.125 \mu \mathrm{g} / \mathrm{ml}$. Derivatives of rifalazil (new chemical entities; NCEs) showed from 2 4 fold lower MICs, as well as $2 \sim 8$ fold lower bactericidal concentrations against both wild type and mutant strains when compared with rifalazil. These results suggest that rifalazil and NCEs are appropriate therapeutic agents for the treatment of C. pneumoniae infections from the point of view of potency and resistance development.
\end{abstract}

Keywords rifalazil, Chlamydia, rifampin, resistance, Chlamydia pneumoniae

\section{Introduction}

Rifalazil [3'-hydroxy-5'-(4-isobutyl-1-piperazinyl) benzoxazinorifamycin] represents a new generation of rifamycins, compounds which inhibit bacterial RNA polymerases isolated from a broad spectrum of microorganisms [1]. Clinically the rifamycins rifampin, rifabutin, and rifapentine have been used predominantly to treat tuberculosis, an indication for which rifalazil (also

D. M. Rothstein (Corresponding author), C. K. Murphy: ActivBiotics, Inc., Lexington, MA, E-mail: drothstein@rcn.com R. J. Suchland, M. Xia, W. E. Stamm: Division of Allergy and Infectious Diseases, Department of Medicine, University of Washington, Seattle, WA known as KRM-1648 and as ABI-1648), was originally developed by Kaneka Corporation, Osaka, Japan, through phase II clinical trials [2, 3].

More recently it has been found that rifalazil was 50 500 times more potent against the obligate intracellular pathogens Chlamydia trachomatis [4, 5], and Chlamydia pneumoniae [4, 6], than azithromycin, the standard of care in treating chlamydial infections. Chlamydia trachomatis is is a leading cause of sexually transmitted disease and of preventable blindness (trachoma) worldwide [8]. Chlamydia pneumoniae is a frequent cause of respiratory infections [9]. Potency may be important to overcome Chlamydia, which can enter the persistent state, which may be more refractory to many antibiotics $[10,11]$. Rifalazil has a long half-life and large volume of distribution, properties that may enhance efficacy against these obligate intracellular pathogens in vivo [3,12,13]. Animal studies support this hypothesis. In the mouse pneumonitis model of infection with $C$. pneumoniae, rifalazil administered as 3 daily doses of $1 \mathrm{mg} / \mathrm{kg}$ was effective in clearing lung infection $[6,7]$.

In a Phase II clinical trial, a single $25 \mathrm{mg}$ oral dose of rifalazil was shown to eradicate $C$. trachomatis from the majority of male patients infected with this bacterium who presented with nongonococcal urethritis [14]. More recently rifalazil has been evaluated for treatment of peripheral arterial disease, based on an association of atherosclerosis and C. pneumoniae infection, supported by animal model studies, and investigations showing the presence of C. pneumoniae in diseased vasculature [13, $15]$.

In this paper we address concerns about the potential for resistance development during rifalazil monotherapy. Both rifalazil and rifampin have been shown to share the property of frequent selection of resistant strains following 
exposure of these drugs to free-living bacteria such as Mycobacterium tuberculosis [16 19], Staphylococcus aureus [20 22], and Streptococcus pyogenes [23]. Mutations in the rроB genes of these microorganisms account for the development of resistance. However, rifalazil was shown previously to be less prone to resistance selection when C. trachomatis was tested in cell culture, even though rifampin resistance was high [24]. The eradication of $C$. trachomatis among patients treated with rifalazil also suggests that resistance development among Chlamydia is not a high frequency consequence of rifalazil monotherapy [14].

In a previous study, mutants of $C$. pneumoniae having low-level resistance to rifampin and rifalazil were isolated, although the same mutants were not tested for susceptibility of both rifampin and rifalazil [25]. To advance these results, an extensive effort was undertaken to isolate high-level rifampin-resistant mutants of $C$. pneumoniae, and to test whether rifalazil and derivative compounds retained activity against these isolates.

\section{Materials and Methods}

\section{Chemicals}

Rifampin, obtained from Sigma, was prepared in DMSO to a concentration of $10 \mathrm{mg} / \mathrm{ml}$ and subsequently diluted in DMSO if necessary, to achieve concentrations ranging from 0.000032 to $0.5 \mu \mathrm{g} / \mathrm{ml}$. Rifalazil and NCEs were provided by ActivBiotics.

\section{Organisms}

Hep-2 mammalian cells were the host. C. pneumoniae strains TW-183 and CWL029 were the susceptible wild type parent strains.

\section{Susceptibility Tests}

The MIC test was as previously described [26]. Briefly, Hep-2 mammalian monolayers were formed following incubation of $100 \mu 1 /$ well in 96-well plates in antimicrobialfree growth medium consisting of minimal essential medium with $10 \%$ fetal bovine serum and $220 \mu \mathrm{g}$ of Lglutamine/liter added. Cells were centrifuged $(1,200 \times g$ for 1 hour at $37^{\circ} \mathrm{C}$ ), and were then exposed to growth medium containing $1.0 \mu \mathrm{g} / \mathrm{ml}$ cycloheximide and two-fold dilutions of rifamycins. After incubation for 72 hours at $37^{\circ} \mathrm{C}$ in $4.0 \% \mathrm{CO}_{2}$, cells were incubated and fixed with $\mathrm{MeOH}$. Chlamydial inclusions were detected by fluorescence microscopy by using a genus-specific monoclonal antibody CF-2 (Washington Research Foundation, Seattle). MCC3 results were determined as described [26], utilizing the same initial procedure as in MIC test, except that three antibiotic-free passages were carried out prior to assaying for inclusions.

\section{Protection Experiments}

The determination of protective concentrations after exposure to rifamycins was as described [27]. Briefly, mammalian cells were treated as in the MIC testing, exposed to rifamycins for 24 hours, centrifuged, washed, and incubated in fresh antibacterial-free medium, until elementary bodies of C. pneumoniae were added either 2 or 7 days of incubation in antibacterial-free medium. Medium was changed every two days when protection was tested after incubation for 7 intervening days.

\section{Selection of Rifampin-resistant Mutants}

If one Chlamydia preparation were the source of all inoculations during mutant selection, it might contain predominantly one rifampin-resistant mutant genotype as a sub-population. To avoid the possibility of selecting repeatedly for the same mutant, and to assure the best chance of isolating mutants from independent genetic events, it was necessary to make several preparations of Chlamydia, each preparation seeded with 1 or a limited number of homogeneous wild type, rifampin-susceptible bacteria (cloning by limiting dilution) [24]. Therefore, as a first step, C. pneumoniae serovar TW-183 was microbiologically cloned by limiting dilution to make 4 preparations. In addition a fifth preparation of another C. pneumoniae variant, CWL-029, was also made.

Chlamydia from each preparation were inoculated at a multiplicity of 5 onto Hep-2 mammalian cell monolayers in 25 -cm flasks, and grown in the presence two-thirds the MIC of rifampin, in a method similar to that used to obtain mutants of C. trachomatis [24]. After 72 hours of incubation, the monolayer was lysed by sonication, and debris removed by slow-speed centrifugation. Aliquots of the supernatant were then inoculated onto fresh monolayers of Hep-2 cells in $8 \sim 12 \mathrm{~mm}$ shell vials, containing increasing amounts of rifampin, starting with a low concentration of $0.0053 \mu \mathrm{g} / \mathrm{ml}$ of rifampin ( $2 / 3$ the MIC) up to the high concentration $0.5 \mu \mathrm{g} / \mathrm{ml}$, as depicted in Fig. 1 .

Growth was monitored on corresponding wells of a 48well plate to observe emerging resistance by routine microscopic immunofluorescent techniques described previously [24]. Plates were stained and read at 72 hours, and vials were passed onto fresh shell vial monolayers and overlaid with previous corresponding rifampin concentrations. After three passages of Chlamydia in shell vials maintaining the same concentration of rifampin in each case, the MIC was determined for both rifampin and 


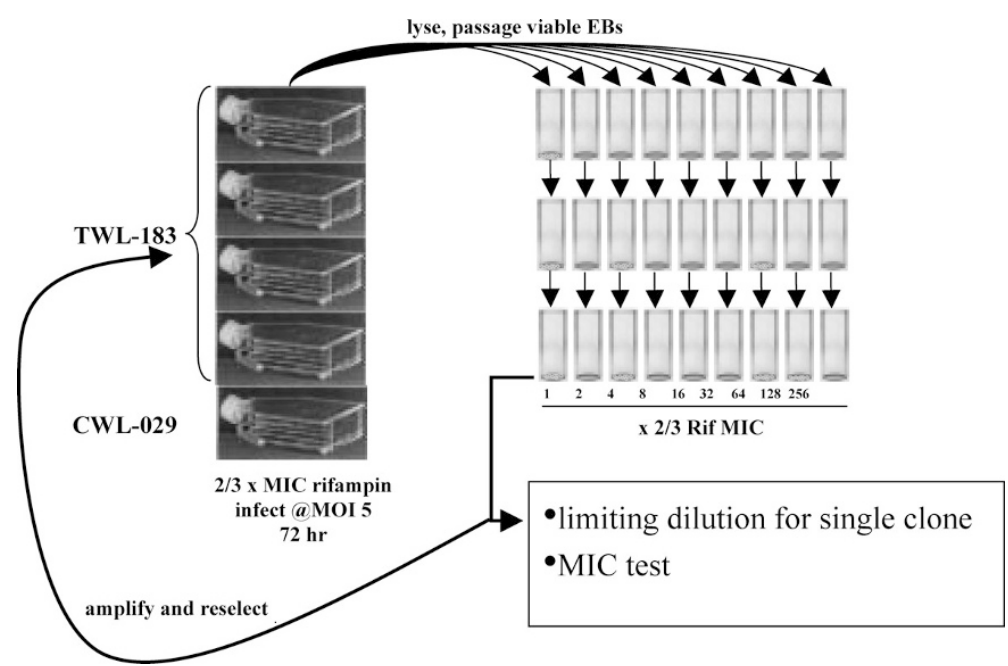

Fig. 1 Illustration of the method of selection of $C$. pneumoniae mutants resistant to rifampin.

C. pneumoniae were inoculated onto Hep-2 mammalian cells in flasks containing rifampin, and inoculated in subsequent passages in shell vials containing varying concentrations of rifampin as shown. Inclusion bodies observed in some vials indicated the presence of mutants. ( $E B=$ elementary body)

rifalazil by testing for inclusion body formation in the presence of 2-fold dilutions of these rifamycins. If the observed MIC exceeded the starting MIC, the mutant was purified and isolated by cloning by limiting dilution.

A portion of the sample containing the mutant(s) was also subjected to selection a second time. This enabled the isolation of a new derivative containing not only the original mutation, but also a second mutation. It was thus possible that these strains with multiple point mutations would be even more resistant to rifampin as seen in a previous study [24]. The method of passage in shell vials was identical this second time through, starting with $2 / 3$ the mutant MIC.

If no emergence of resistance was observed in a particular sample after three passages, the selection regimen was repeated, in order to provide a second opportunity of recovering mutants from the wild type preparations.

\section{DNA Sequenceing}

To define mutations in the C. pneumoniae rpoB, we sequenced PCR products covering nucleotides 1235 through 1785, corresponding to amino acid 412 through 595 for mutants and control strains. PCR products of this segment were amplified with primers designed based on the rроB (Cpn0081) sequence of reference strain CWL029 using high fidelity DNA polymerase (PfuUltra, Stratagene). Both the sense and anti-sense strands were sequenced three times using forward primer CPrpoBF1: 5'-ATCTTGCTAATCGACGTGTCC and reverse primer CPrpoBR: 5'-
ACATTCTAATCCAGTGCCAAC. Consensus sequences were derived from assembled contigs and aligned with the Sequencher program.

\section{Results}

\section{Characterization of Mutants by DNA Sequencing}

From two of the four preparations of TW-183, no mutants were isolated. From the other two preparations of TW-183, as well as one preparation of CWL029, four mutants were isolated containing different single mutations, and 3 different mutants defined by having two mutations, all within the RNA polymerase $\beta$ subunit gene, encoding the rifamycin binding site. Therefore, rifampin resistance was explained in each mutant phenotype by an alteration in the subunit of RNA polymerase containing the rifampin binding site.

The locations of mutations leading to rifamycin resistance, defined by DNA sequencing, are shown relative to mutations found in other species (Fig. 2). In the four cases of single mutations, all alterations were in residues at or near known contact points between rifampin and RNA polymerase from crystal structure studies with the RNA polymerase purified from the bacterium Thermos aquatis [28]. The mutation at position 456, in fact, is in the same position as the mutation described in the previous study [25]. Codon 478 may also be a hotspot for mutations in C. pneumoniae, because mutants were independently isolated containing the same mutation at 478 from two 


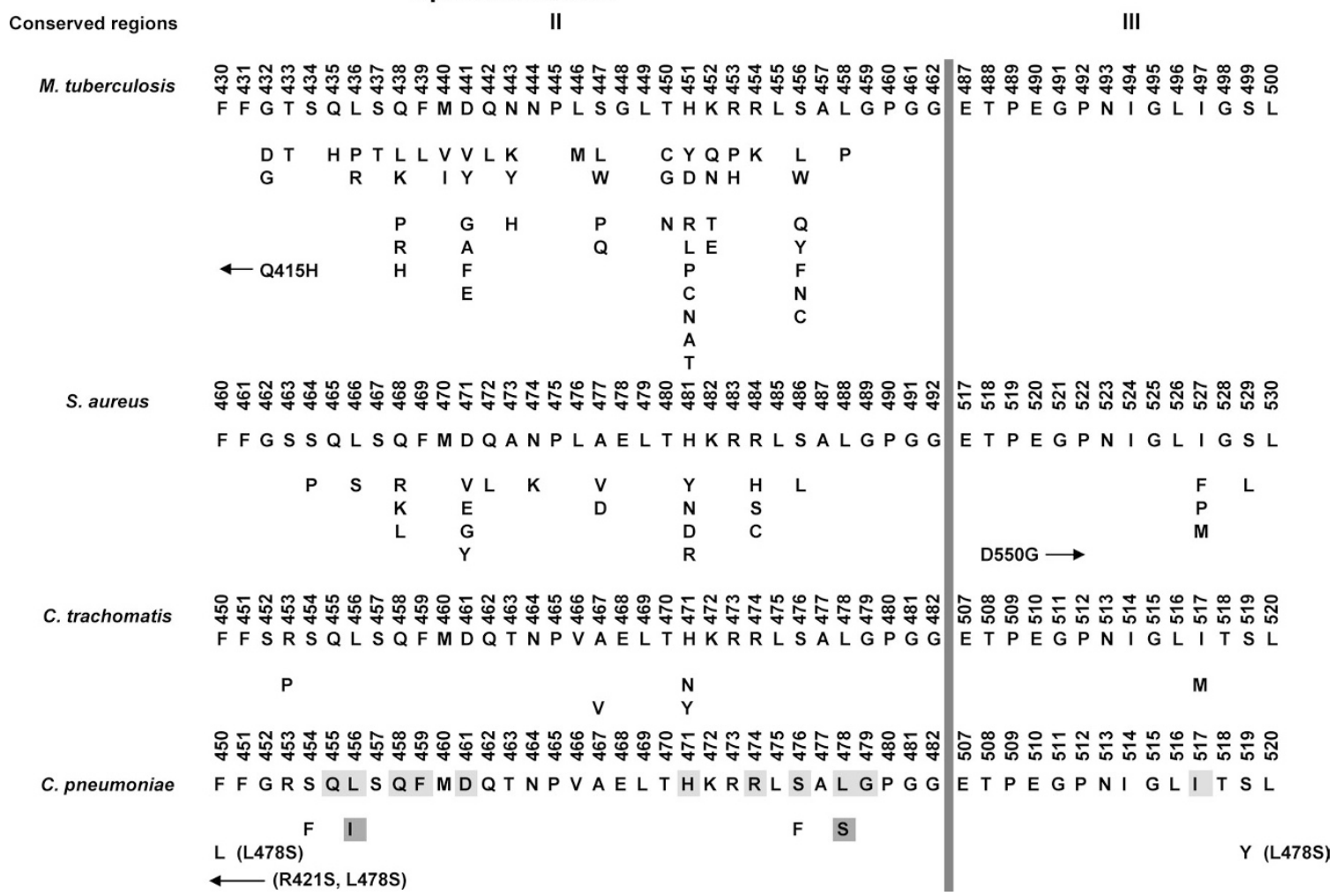

Fig. 2 Mutations in the rpoB gene from C. pneumoniae rifampin-resistant mutants isolated in this report.

The sequences of the rpoB genes and rifampin-resistant mutants of Mycobacterium tuberculosis, Staphylococcus aureus, Chlamydia trachomatis, in addition to $C$. pneumoniae, are shown for comparison. The residues known to interact with rifampin by crystallographic analysis of RNA polymerase from Thermos aquatis [2] are shaded. When mutations of $C$. pneumoniae are found only in combination, the second mutation is denoted on the same line in parenthesis.

Table 1 Rifampin and rifalazil MICs $(\mu \mathrm{g} / \mathrm{ml})$ against wild type and mutant strains of $C$. pneumoniae

\begin{tabular}{|c|c|c|c|c|c|c|}
\hline Parent & Mutation & Codon change & MIC/Rifampin & MIC/Rifalazil & $\begin{array}{l}\text { X MIC wt } \\
\text { Rifampin* }\end{array}$ & $\begin{array}{l}\text { X MIC wt } \\
\text { Rifalazil* }\end{array}$ \\
\hline TW-183 wild type & None & Wild-type & 0.008 & 0.00025 & - & - \\
\hline CWL-029 wild type & None & Wild-type & 0.008 & 0.00025 & - & - \\
\hline TW-183 & TCC->TTC & S454F & 0.032 & 0.00025 & 4 & 1 \\
\hline CWL-029 & TCT->TTT & Ł456। & 0.125 & 0.0005 & 16 & 2 \\
\hline TW-183 & TTA->TCA & L478S & 2 & 0.004 & 256 & 16 \\
\hline TW-183 & TTA->TCA, TTC->TTA & L478S F450S & 4 & 0.008 & 512 & 32 \\
\hline TW-183 & TTA->TCA, AGA->AGC & L478S R421S & 32 & 0.064 & 4096 & 256 \\
\hline TW-183 & TTA->TCA, TCT->TAT & L478S S519Y & 32 & 0.064 & 4096 & 256 \\
\hline CWL-029 & TCT->TTT & S476F & 128 & 0.125 & 16384 & 512 \\
\hline
\end{tabular}

* The fold increase in MIC compared to the wild type MIC for mutant strains.

different wild type preparations, and this mutation was also found in the three mutants carrying two mutations.
Characterization of Mutants by Susceptibility Testing in Cell Culture

The MIC results shown in Table 1 clearly show that the 
mutants were considerably more resistant to rifampin than to rifalazil. Thus, the MIC for rifampin was very high (above $100 \mu \mathrm{g} / \mathrm{ml}$ for the most resistant mutant), a concentration that is unachievable in therapy under normal physiological conditions. The rifalazil MIC for even the highest mutant was $0.125 \mu \mathrm{g} / \mathrm{ml}$. Considering the high intracellular level of rifalazil in mammalian cells [13], an inhibitory concentration of rifalazil may be achievable during clinical use. Thus the physiological concentrations of rifalazil, but not of rifampin, may be sufficient to reduce the selection of mutants.

The second method of comparison between rifampin and rifalazil is calculated by considering the MICs of wild type cells as the baseline level of potency, and to determine the increase in MICs of mutants relative to the MIC against the wild type parent strain. By this measure, rifalazil had a considerably lower ratio of mutant to wild type MIC (512 times the wild type MIC for the most resistant mutant) than did rifampin (16,384 times the wild type MIC) (Table 1).

\section{Activity of NCEs}

The potency of NCEs ABI-0043, ABI-0369, ABI-0597, and ABI-0699, depicted in Fig. 3, are shown in Table 2. The MIC of all the NCEs was $2 \sim 4$ fold lower than that of rifalazil. This difference was also noted in the determination of the minimal bactericidal concentration (MCC3; the concentration sufficient to suppress any growth following exposure and 3 subsequent passages in antibacterial-free medium). The NCEs had $2 \sim 8$ fold lower

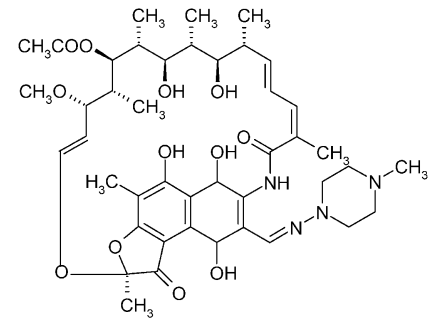

\begin{tabular}{|l|l|c|}
\hline Compound & $\mathrm{R}^{1}$ & $\mathrm{R}^{2}$ \\
\hline Rifalazil & $\mathrm{OH}$ & \\
\hline $\mathrm{ABI}-0043$ & $\mathrm{OH}$ & \\
\hline $\mathrm{ABI}-0369$ & $\mathrm{O}-\mathrm{AC}$ & \\
\hline $\mathrm{ABI}-0699$ & $\mathrm{O}-\mathrm{Ac}$ & \\
\hline
\end{tabular}

$* \mathrm{OH}=$ hydroxyl; $\mathrm{O}-\mathrm{Ac}=\mathrm{O}$-acetyl

Fig. 3 Structures of rifamycins.

Table 2 Activities of NCEs compared with rifalazil in cell culture

\begin{tabular}{cllllll}
\hline Strain & Test & Rifalazil & ABI-0043 & ABI-0369 & ABI-0597 & ABI-0699 \\
\hline \multirow{2}{*}{ TW-183 } & MIC & 0.00025 & 0.000125 & 0.000125 & 0.000064 & 0.000064 \\
(wild type) & MCC & 0.008 & 0.002 & 0.004 & 0.001 & 0.001 \\
& PD2 & 0.016 & 0.008 & 0.008 & 0.002 & 0.002 \\
& PD7 & 0.032 & 0.016 & 0.016 & 0.004 & 0.004 \\
L456I & MIC & 0.0005 & 0.0005 & 0.00025 & 0.00025 & 0.00025 \\
& MCC & 0.016 & 0.008 & 0.004 & 0.002 & 0.002 \\
& PD2 & 0.032 & 0.016 & 0.002 & 0.004 & 0.008 \\
L478S & PD7 & 0.064 & 0.032 & 0.008 & 0.016 & 0.064 \\
& MIC & 0.008 & 0.002 & 0.001 & 0.001 & 0.001 \\
& MCC 3 & 0.125 & 0.016 & 0.016 & 0.008 & 0.016 \\
S476F & PD2 & 0.125 & 0.064 & 0.016 & 0.032 & 0.064 \\
& PD7 & 0.25 & 0.125 & 0.016 & 0.064 & 0.25 \\
& MIC & 0.125 & 0.032 & 0.032 & 0.032 & 0.032 \\
& MCC 3 & $>5$ & 1 & 1 & 0.5 & 0.5 \\
& PD2 & 5 & 0.5 & 0.5 & 0.25 & 0.5 \\
\hline
\end{tabular}

${ }^{*} \mathrm{MIC}=$ Minimal inhibitory concentration, $\mathrm{MCC}_{3}=$ minimal bactericidal concentration, PD2 and PD5=Protective Dose after 2 and 5 days, respectively. 
$\mathrm{MCC}_{3}$ s compared with rifalazil. The protective concentration was determined by exposing mammalian cells to the indicated compounds, and then growing the mammalian cells for either two or seven subsequent days of incubation in antibacterial-free medium prior to infection, as previously described [27]. The antibacterial concentration affording protection was again $2 \sim 8$ fold lower for NCEs than for rifalazil.

\section{Discussion}

The selection and characterization of mutants in this paper that are highly resistant to rifampin demonstrates that rifalazil is less prone to development of resistance. The highest MIC against $C$. pneumoniae mutants was only $0.125 \mu \mathrm{g} / \mathrm{ml}$, compared to an MIC of $>100 \mu \mathrm{g} / \mathrm{ml}$ for rifampin. Thus it is possible that rifalazil would not select for resistant mutants of $C$. pneumoniae during monotherapy. These results are consistent with those found previously when the same question was examined in the case of the related obligate intracellular pathogen, $C$. trachomatis, in which the highest rifalazil MIC was $0.0064 \mu \mathrm{g} / \mathrm{ml}$, despite the isolation of mutants fully resistant to rifampin [24]. In considering these results, together with those obtained for $C$. trachomatis [24, 25], it is clear that Chlamydia presents a special case in which rifalazil is not only potent against wild type bacteria, but also retains activity against mutant strains having chromosomal mutations in the $r p o B$ gene which result in strong resistance to rifampin. The fact that rifalazil as a monotherapeutic agent was at least as effective as azithromycin, the standard of care, in the microbiological cure of $C$. trachomatis among men having nongonococcal urethritis in a clinical trial further supports the idea that development of resistance to rifalazil by Chlamydia is not a frequent event [14].

A total of 7 distinct rifampin-resistant mutants of C. pneumoniae, four strains having unique single mutations, and 3 strains having a unique second mutation in combination with the primary mutation at codon position 456 , were isolated. It is interesting that the identical $\mathrm{C} \rightarrow \mathrm{A}$ transversion resulting in the codon change, L478S, was found in several samples derived from TW-183 preparation 1 , and in TW-183 preparation 3. In addition, one mutant isolated from strain CPL029 contained an identical mutation to an allele reported previously [25], conferring the L456I change in RpoB. Even with the occurrence of these repeated genetic events, which might suggest mutational hotspots in the rроB gene, rifampin-resistant mutants of $C$. pneumoniae were less frequent than for mutants derived from $C$. trachomatis characterized previously [24]. It is possible that $C$. pneumoniae is less prone to rifampin-resistance development than $C$. trachmatis. Alternatively, it is also possible that less growth occurred in the case of the slower growing $C$. pneumoniae, and that fewer elementary bodies were transferred after each passage, which could account for the lower frequency of mutants isolated.

The NCEs that were tested were even more active than rifalazil in cell culture tests. The lower MICs of NCEs ABI0043, ABI-0369, ABI-0597, and ABI-0699 (Table 2) is in keeping with previous findings that some NCEs are more active than rifalazil against $C$. trachomatis against both wild type and rifampin-resistant strains [29]. The NCEs also had lower $\mathrm{MCC}_{3} \mathrm{~s}$ (bactericidal concentrations) against C. pneumoniae than rifalazil by $2 \sim 8$ fold (Table 2 ), further suggesting that NCEs have potential that could even surpass that of rifalazil in treating chlamydial infections. The NCEs also had a greater protective effect than rifalazil, showing that these derivative compounds share with rifalazil the propensity to concentrate and remain inside mammalian cells, and to exert their antichlamydial effect days after exposure. This attribute of rifalazil and the NCEs may prove to be valuable in eradicating Chlamydia and preventing it from persisting inside of host cells.

\section{References}

1. Fujii K, Saito H, Tomioka H, Mae T, Hosoe K. Mechanism of action of antimycobacterial activity of the new benzoxazinorifamycin KRM-1648. Antimicrob Agents Chemother 39: 1489-1492 (1995)

2. Dietze R., Teixeira L, Rocha LM, Palaci M, Johnson JL, Wells C, Rose L, Eisenach K, Ellner JJ. Safety and bactericidal activity of rifalazil in patients with pulmonary tuberculosis. Antimicrob Agents Chemother 45: 1972-1976 (2001)

3. Rothstein DM, Hartman AD, Cynamon MH, Eisenstein BI. Development potential of rifalazil. Expert Opin Investig Drugs 12: 255-271 (2003).

4. Roblin PM, Reznik T, Kutlin A, Hammerschlag MR. In vitro activities of rifamycin derivatives ABI-1648 (rifalazil, KRM-1648), ABI-1657, and ABI-1131 against Chlamydia trachomatis and recent clinical isolates of Chlamydia pneumoniae. Antimicrob Agents Chemother 47: 1135-1136 (2003)

5. Stamm WE, Geisler WM, Suchland RJ. Assessment of antimicrobial resistance in Chlamydia trachomatis strains associated with treatment failure or same strain recurrence, pp. 353-360. In Chlamydial infections: Proceedings of the 10th International Symposium on Human Chlamydial Infections. Basim Yeri: GRAFMAT Basim ve Reklam 
Sanayi Tic. Ltd. Sti., Antalya, Turkey (2002)

6. Kuo C-C, Grayston JT, Hidaka T, Rose LM. A comparison of the in vitro sensitivity of Chlamydia pneumoniae to macrolides and a new benzoxazinorifamycin, KRM-1648. In Expanding indications for the new macrolides, azalides, and streptogramins. Infectious disease and therapy series, Vol. 21, Ed., Zinner SH, et al., pp. 317-321, Marcel Dekker, New York, N.Y. (1997)

7. Campbell LA, Kuo CC, Suchland RJ, Rothstein DM. Efficacy of benzoxazinorifamycins in a mouse model of Chlamydia pneumoniae lung infection. Antimicrob Agents Chemother 52: 1855-1858 (2008)

8. Schachter J, West SK, Mabey D, Dawson CR, Bobo L, Bailey R, Vitale S, Quinn TC, Sheta A, Sallam S, Mkocha H, Mabey D, Faal H. Azithromycin in control of trachoma. Lancet 354: 630-635 (1999)

9. Grayston JT. Chlamydia pneumoniae, strain TWAR pneumonia. Annu Rev Med 43: 317-323 (1992)

10. Beatty WL, Morrison RP, Byrne GI. Persistent chlamydiae: from cell culture to a paradigm for chlamydial pathogenesis. Microbiol Rev 58: 686-699 (1994)

11. Ouellette SP, Hatch TP, AbdelRahman YM, Rose LA, Belland RJ, Byrne GI. Global transcriptional upregulation in the absence of increased translation in Chlamydia during IFNgamma-mediated host cell tryptophan starvation. Mol Microbiol 62: 1387-1401 (2006)

12. Hosoe K, Mae T, Konishi E, Fujii K, Yamashita K, Yamane T, Hidaka T, Ohashi T. Pharmacokinetics of KRM-1648, a new benzoxazinorifamycin, in rats and dogs. Antimicrob Agents Chemother 40: 2749-2755 (1996)

13. Rothstein DM, Shalish C, Murphy CK, Sternlicht A, Campbell LA. Development potential of rifalazil and other benzoxazinorifamycins. Expert Opin Investig Drugs 15: 603-623 (2006)

14. Stamm WE, Batteiger BE, McCormack WM, Totten PA, Sternlicht A, Kivel NM. For the Rifalazil Study Group. A Randomized, Double-Blind Study Comparing Single-Dose Rifalazil With Single-Dose Azithromycin for the Empirical Treatment of Nongonococcal Urethritis in Men. Sex Transm Dis. Feb 8; [Epub ahead of print] (2007)

15. Rothstein DM, van Duzer J, Sternlicht A, Gilman SC. Rifalazil and other benzoxazinorifamycins in the treatment of Chlamydia-based persistent infections. Arch Pharm (Weinheim) 340: 517-529 (2007)

16. Moghazeh SL, Pan X, Arain T, Stover CK, Musser JM, Kreiswirth BN. Comparative antimycobacterial activities of rifampin, rifapentine, and KRM-1648 against a collection of rifampin-resistant Mycobacterium tuberculosis isolates with known rpoB mutations. Antimicrob Agents Chemother 40: 2655-2657 (1996)

17. Williams DL, Spring L, Collins L, Miller LP, Heifets LB, Gangadharam PR, Gillis TP. Contribution of $r p o B$ mutations to development of rifamycin cross-resistance in Mycobacterium tuberculosis. Antimicrob Agents Chemother 42: 1853-1857 (1998)
18. Yang B, Koga H, Ohno H, Ogawa K, Fukuda M, Hirakata Y, Maesaki S, Tomono K, Tashiro T, Kohno S. Relationship between antimycobacterial activities of rifampicin, rifabutin and KRM-1648 and rpoB mutations of Mycobacterium tuberculosis. J Antimicrob Chemother 42: 621-628 (1998)

19. Park YK, Kim BJ, Ryu S, Kook YH, Choe YK, Bai GH, Kim SJ. Cross-resistance between rifampicin and KRM-1648 is associated with specific rpoB alleles in Mycobacterium tuberculosis. Int $\mathrm{J}$ Tuberc Lung Dis 6: 166-170 (2002)

20. Wichelhaus TA, Schafer V, Brade V, Boddinghaus B. Molecular characterization of rpoB mutations conferring cross-resistance to rifamycins on methicillin-resistant Staphylococcus aureus. Antimicrob Agents Chemother 43: 2813-2816 (1999)

21. Wichelhaus TA, Schafer V, Brade V, Boddinghaus B. Differential effect of $r p o B$ mutations on antibacterial activities of rifampicin and KRM-1648 against Staphylococcus aureus. J Antimicrob Chemother 47: 153-156 (2001)

22. Murphy CK, Mullin S, Osburne MS, van Duzer J, Siedlecki J, Yu X, Kerstein K, Cynamon M, Rothstein DM. In vitro activity of novel rifamycins against rifamycin-resistant Staphylococcus aureus. Antimicro Agents Chemother 50: 827-834 (2006)

23. Mullin S, Rothstein DM, Murphy CK. Activity of novel benzoxazinorifamycins against rifamycin-resistant Streptococcus pyogenes. Antimicrob Agents Chemother 50: 1908-1911 (2006)

24. Suchland RJ, Bourillon A, Denamur E, Stamm WE, Rothstein DM. Rifampin-resistant RNA polymerase mutants of Chlamydia trachomatis remain susceptible to the ansamycin rifalazil. Antimicrob Agents Chemother 49: 1120-1126 (2005)

25. Kutlin A, Kohlhoff S, Roblin P, Hammerschlag MR, Riska P. Emergence of resistance to rifampin and rifalazil in Chlamydophila pneumoniae and Chlamydia trachomatis. Antimicrob Agents Chemother. 49: 903-907 (2005)

26. Suchland RJ, Geisler WM, Stamm WE. Methodologies and cell lines used for antimicrobial susceptibility testing of Chlamydia spp. Antimicrob Agents Chemother 47: 636-642 (2003)

27. Suchland RJ, Brown K, Rothstein DM, Stamm WE. Rifalazil pretreatment of mammalian cell cultures prevents subsequent Chlamydia infection. Antimicrob Agents Chemother 50: 439-444 (2006)

28. Campbell EA, Korzheva N, Mustaev A, Murakami K, Nair S, Goldfarb A, Darst SA. Structural mechanism for rifampicin inhibition of bacterial RNA polymerase. Cell 104: 901-912 (2001)

29. Xia M, Suchland RJ, Carswell JA, Van Duzer J, Buxton DK, Brown K, Rothstein DM, Stamm WE. Activities of rifamycin derivatives against wild-type and $r p o B$ mutants of Chlamydia trachomatis. Antimicrob Agents Chemother 49: 3974-3976 (2005) 